\title{
Avaliação da Leptina em Pacientes Portadoras da Sindrome dos Ovários Policisticos - Estudo de Suas Relações com a Testosterona, 0 Estradiol, o FSH e a Insulina
}

\author{
Autor: Marco Antônio Barreto de Melo
}

Orientador: Prof. Dr. Selmo Geber

Tese de Mestrado apresentada ao Departamento de Pós-graduação de Ginecologia e Obstetrícia da Faculdade de Medicina da Universidade Federal de Minas Gerais, em 16 de fevereiro de 2001.

Objetivos: a leptina é uma proteína secretada pelos adipócitos e que participa na regulação do peso. Mulheres portadoras da sindrome dos ovários policísticos (SOP) são freqüentemente obesas, insulino-resistentes, hiperinsulinêmicas e inférteis. Desta forma, realizamos este estudo com o objetivo de verificar a presença de uma relação entre a leptina e SOP.

Pacientes e Métodos: desenvolvemos um estudo transversal com 40 pacientes portadoras de SOP, divididas de acordo com o índice de massa corporal (IMC): Grupo I ( $\mathrm{n}=20)$ - pacientes obesas (IMC $\left.\geq 28 \mathrm{~kg} / \mathrm{m}^{2}\right)$ e Grupo II $(\mathrm{n}=20)$ - pacientes não-obesas $\left(\mathrm{IMC}<28 \mathrm{~kg} / \mathrm{m}^{2}\right)$. Foi realizada análise de regressão entre a leptina, a insulina, a relação glicemia/insulina, o estradiol, os androgênios e o FSH.

Resultados: o IMC foi diferente estatisticamente entre os 2 grupos $(p=0,04)$. Verificamos uma diferença, com tendência à significância estatística, entre os níveis de insulina das pacientes não obesas e obesas $(p=0,05)$. Foi observada significância estatística entre a relação glicemia/insulina e os grupos $(p=0,043)$. Os níveis de leptina se mostram fortemente correlacionados com o IMC $(p<0,001)$. Verificou-se, após a regressão, eliminando-se o efeito do IMC, apenas uma correlação entre as concentrações da leptina e do estradiol $(p=0,043)$, sendo constatado que existe um aumento de 0,054 ng/ $\mathrm{mL}$ nos niveis de leptina para cada aumento de $1,0 \mathrm{ng} /$ dL na dosagem de estradiol.

Conclusão: Podemos concluir que existe uma forte correlação entre os niveis de leptina, o IMC e as concentrações de estradiol, em mulheres portadoras de SOP, sem, entretanto, haver participação na sua patogênese.

Palavras-chave: Síndrome dos ovários policísticos. Leptina. Obesidade. Infertilidade. Anovulação.

\section{Estudo da Cardiotocografia Anteparto em Gestaçoess com Diástole Zero ou Reversa à Dopplervelocimetria das Artérias Umbilicais}

Autora: Roseli Mieko Yamamoto Nomura

Orientador: Prof. Dr. Seizo Miyadahira

Tese apresentada à Faculdade de Medicina da Universidade de São Paulo, para obtenção do Título de Doutor em Medicina, em 7 de fevereiro de 2001.

Cento e vinte e sete gestações com diástole zero ou reversa à dopplervelocimetria das artérias umbilicais foram analisadas retrospectivamente, com o objetivo de se determinar a relação entre a cardiotocografia anteparto de repouso e a ocorrência de acidemia no nascimento, comparando-se as características da freqüência cardiaca fetal com os resultados neonatais. Os seguintes parâmetros foram estudados: freqüência cardiaca fetal basal, variabilidade, acelerações transitórias, padrão sinusoidal, desacelerações tardias, variáveis e prolongadas. A análise multivariada pelo modelo de regressão logística demonstrou que: a variabilidade inferior a cinco batimentos por minuto, a presença de desacelerações tardias e a ocorrência de desacelerações variáveis do tipo grave relacionaramse com a ocorrência de valores inferiores a 7,20 no $\mathrm{pH}$ arterial de cordão umbilical no nascimento; e a variabilidade inferior a cinco batimentos por minuto e a pre- sença de desacelerações tardias relacionaram-se com a ocorrência de valores inferiores a 7,15 no $\mathrm{pH}$ arterial de cordão umbilical no nascimento. Este estudo permitiu elaborar modelo matemático capaz de estimar a probabilidade da ocorrência de acidemia no nascimento, a partir do conhecimento destes parâmetros cardiotocográficos no periodo anteparto, em gestações com diástole zero ou reversa das artérias umbilicais. A observação de parâmetros anormais na cardiotocografia associaram-se significativamente com: menor idade gestacional no nascimento, recém-nascidos de menor peso, menores valores no Apgar de $1^{\circ}$ minuto, intubação do recém-nascido, óbito neonatal precoce e óbitos no berçário.

Palavras-chave: Cardiotocografia. Monitorização Fetal. Ultra-sonografia Doppler. Vitalidade Fetal. Freqüência cardiaca fetal. 


\section{Avaliação do Parceiro Sexual e Risco de Recidivas em Mulheres Tratadas por Lesões Genitais Induzidas por Papilomavirus Humano(HPV)}

Autor: Júlio César Teixeira

Orientador: Prof. Dr. Luiz Carlos Teixeira

Tese apresentada ao Departamento de Tocoginecologia da Faculdade de Ciências Médicas da Universidade Estadual de Campinas para obtenção do Título de Doutor em Medicina, Área de Tocoginecologia, em 15 de dezembro de 2000.

Objetivo: estudar a associação entre a avaliação ou não do parceiro de mulheres portadoras de lesões genitais HPV-induzidas e a ocorrência de recidivas nestas mulheres.

Métodos: estudo de coorte reconstituído com $144 \mathrm{mu}-$ lheres portadoras de lesões e cujos parceiros foram avaliados, comparadas com 288 mulheres controladas por data do atendimento, idade, grau das lesões e tratamento, mas cujos parceiros não foram examinados, atendidos na UNICAMP, entre julho/1993 e março/ 2000. Avaliou-se a semelhança entre os grupos, a associação entre a avaliação ou não dos parceiros ou o diagnóstico de lesões HPV-induzidas, com a ocorrência e grau das recidivas nas mulheres e o tempo livre de doença (TLD).

Resultados: os grupos foram semelhantes na idade, início sexual, tempo de atividade sexual, número de parceiros, tabagismo, grau da lesão e tratamento realizado. Encontrou-se 9,0\% de recidivas nas mulheres cujos parceiros foram avaliados contra 5,9\% quando os parceiros não foram examinados $(p=0,23)$. Quando se diagnosticou lesões nos homens, observou-se 1,5 vezes mais recidivas nas suas parceiras $(12,5 \%$ contra $7,3 \%, p=0,23)$, mas sem correlação com o grau da lesão recidivada e TLD. Quando o homem relatou estabilidade conjugal $\leq 12$ meses, observou-se duas vezes mais recidivas $(14,9 \%$ contra $6,2 \%, \mathrm{p}=0,08)$.

Conclusões: a avaliação do homem não diminuiu o risco de recidivas de lesões por HPV na parceira. A presença de lesões nos parceiros não se correlacionou com a ocorrência e grau das recidivas nas mulheres e TLD. Estas observações não suportam a hipótese de que os homens não avaliados seriam uma importante causa de recidivas nas parceiras.

Palavras-chave: HPV. Doenças sexualmente transmissiveis.

RBCO 23(5):334-335, 2001

Resumo de Tese

\section{Análise de Variáveis Maternas e Perinatais em Pacientes com Diagnóstico Histológico Renal de Hipertensão Arterial Crônica e Hipertensão Arterial Crônica com Pré-Eclâmpsia Superajuntada}

Autora: Ione Rodrigues Brum

Orientador: Prof. Dr. Soubhi Kahhale

Tese de Doutorado apresentada à Faculdade de Medicina da Universidade de São Paulo - Clínica Obstétrica da Faculdade de Medicina da USP - São Paulo, em 3 de dezembro de 1999.

Introdução: a hipertensão arterial é uma doença com grande incidência em todo mundo e uma das complicações mais comuns durante a gestação. A incidência de hipertensão arterial crônica na gravidez é maior do que se imagina e um dos motivos é a dificuldade na realização do diagnóstico diferencial entre as diferentes formas de hipertensão da gravidez. Um diagnóstico correto permite um tratamento adequado e desta forma uma diminuição na morbidade e mortalidade materna e perinatal.

Objetivos: a partir do diagnóstico histopatológico de hipertensão arterial crônica e hipertensão arterial crô- nica com pré-eclâmpsia superajuntada obtido por biópsia renal, correlacioná-lo com achados clínicos e laboratoriais maternos e perinatais, identificando o exame clinico e ou laboratorial que permitisse fazer o diagnóstico diferencial entre estas duas doenças.

Casuística e Método: analisamos 96 prontuários de pacientes cujo resultado histopatológico da biópsia renal realizada no pós-parto por indicação de gravidade do quadro hipertensivo foram anatomopatológicos compativeis com hipertensão arterial crônica (60 casos GRUPO I) e anatomopatológicos compativeis com hipertensão arterial crônica mas que apresentavam tam- 
bém endoteliose capilar glomerular (36 casos - GRUPO II).

Resultados: a correlação feita a partir do resultado histopatológico renal com os achados clínicos/ laboratoriais e resultados perinatais não nos permitiu identificar nenhum parâmetro que pudesse ser usado para fazer o diagnóstico diferencial entre as duas formas de hipertensão na gravidez. Contudo observamos nos dois grupos alta incidência de resultado fetal desfavorável com natimortalidade elevada, baixo peso, prematuridade e, ainda, mais de $70 \%$ de cesarianas cujas causas foram sofrimento fetal e piora do quadro materno. Nos chamou especial atenção quanto aos resultados obtidos por este trabalho foi a discordância entre o diagnóstico clínico (de internação) com a histologia renal que ocorreu em mais de $50 \%$ dos casos. Este fato pode sugerir os graus diferentes com que a pré-eclâmpsia acomete os diversos órgãos não sendo portanto inegável a presença de pré-eclâmpsia com a ausência de endoteliose capilar glomerular.

Palavras-chave: Pré-eclâmpsia. Hipertensão arterial. Mortalidade perinatal. Prematuridade.

\section{Alteração Hemodinâmica Fetal, Frente ao Estímulo Vibroacústico, Avaliada através do Método Doppler, ao longo da Gestação}

Autora: Ana Cristina Pinheiro Fernandes Araujo

Orientador: Prof. Dr. Francisco Mauad Filho

Tese de Doutorado apresentada ao Departamento de Ginecologia e Obstetrícia da Faculdade de Medicina de Ribeirão Preto da Universidade de São Paulo em 17 abril de 2000.

Com o intuito de avaliar as possiveis repercussões hemodinâmicas, em nível da artéria cerebral média fetal, após o estímulo sonoro ao longo da gestação, foram analisadas noventa e seis gestantes, consideradas clinicamente normais, atendidas na Clínica Obstétrica (Ambulatório de Pré-Natal) do Departamento de Ginecologia e Obstetrícia da Faculdade de Medicina de Ribeirão Preto, na Escola de Ultra-Sonografia e Reciclagem Médica de Ribeirão Preto e na Maternidade Escola Januário Cicco, da Universidade Federal do Rio Grande do Norte. Analisou-se a artéria cerebral média por meio do método Doppler, ao longo da gravidez, a partir da $26^{\mathrm{a}}$ semana de gestação, até a $40^{\mathrm{a}}$ semana. Compararam-se os resultados obtidos antes e após um estímulo vibroacústico, aplicado durante aproximadamente três a cinco segundos, com uma buzina da marca Kobo. Para análise estatística dos resultados, utilizou-se o teste não paramétrico de Wilcoxon, com nivel de significância de $(\mathrm{p}<0,05)$, e ainda os testes de Kruskal-Wallis, e comparações múltiplas de Dunn. Na análise dos resultados, observou-se diferença, estatisticamente significativa, no índice de resistência da artéria cerebral média, nos fetos a partir da 34a semana até a 40ª semana (neste último grupo houve diferença apenas naquelas com apresentação fetal não insinuada). No grupo de pacientes com idade gestacional entre a 34a e a 36 a semana e 36 e a $38^{\text {a }}$ semana, o indice de resistência mostrou diferença $(\mathrm{p}<0,01)$, e no grupo a termo com apresentação fetal não-insinuada $(\mathrm{p}<0,0068)$. Nos demais grupos, não foi encontrada diferença estatisticamente significativa. Para o indice de pulsatilidade observou-se diferença, estatisticamente significativa, apenas no grupo de gestantes entre a $36^{\mathrm{a}}$ e a $38^{\mathrm{a}}$ semana de gestação. Avaliou-se, ainda, o efeito da idade gestacional sobre os indices de pulsatilidade e resistência, registrando-se diferença em pelo menos dois desses grupos (teste de Kruskal-Wallis). Aplicando-se o teste de comparações múltiplas de Dunn, encontrou-se diferença significativa entre as medianas de ambos os indices entre a $28^{\text {a }}$ a $30^{\text {a }}$ semana e $38^{\mathrm{a}}$ a $40^{\mathrm{a}}$ semana gestacionais. Percebeu-se que o indice de resistência apresenta uma curva decrescente ao longo da gestação. As alterações vasculares do cérebro fetal estão diretamente relacionadas a estímulos físicos. Ante tais repercussões na hemodinâmica vascular cerebral fetal, pôde-se concluir que as alterações do exame Doppler em resposta ao estímulo vibroacústico ocorrem após a 34aㅗ semana, idade em que provavelmente ocorre maturidade neurológica, e que se reflete sobre os mecanismos adaptativos do feto. Constatou-se que esse altera a reatividade vascular não só em estados de hipóxia, mas também como resposta a estímulos físicos externos. A pesquisa desperta novos interesses na área científica, uma vez que estabelece comportamento em fetos hígidos.

Palavras-chave: Vitalidade fetal. Dopplervelocimetria. Gravidez normal. 


\title{
0 Papel do Hormônio do Crescimento, da Insulina e dos Fatores de Crescimento na Fisiopatologia da Sindrome dos Ovários Policísticos
}

\author{
Autora: Ana Cristina Gomes Premoli \\ Orientadora: Profa. Dra. Rosana Maria dos Reis
}

Dissertação de Mestrado apresentada ao Departamento de Ginecologia e Obstetrícia da Faculdade de Medicina de Ribeirão Preto da Universidade de São Paulo, em 15 de maio de 2000.

A Síndrome dos Ovários Policísticos (SOP) é uma doença endócrina multifatorial, apresentando distúrbios do ciclo menstrual (oligo/amenorréia), obesidade, hirsutismo e infertilidade; altos niveis séricos de Hormônio Luteinizante (LH) e elevada relação LH/FSH (Hormônio Folículo Estimulante). À ultra-sonografia evidenciam-se múltiplos cistos nos ovários, demonstrando grande recrutamento folicular, porém sem seleção de um folículo dominante, resultando em anovulação crônica e hiperandrogenismo. A hiperinsulinemia foi evidenciada como co-fator na SOP estando relacionada a elevado índice de massa corporal (IMC). A obesidade contribui na gênese da resistência à insulina (RI), agravando-a. Ocorre também uma diminuição na secreção de Hormônio do Crescimento $(\mathrm{GH})$ e esteroidogênese anormal com altos niveis séricos de LH. O GH na SOP sem obesidade encontra elevação do pulso sérico. Assim, é possivel relacionar RI a pacientes obesas, e ação conjunta do $\mathrm{GH}$ com o LH a mulheres não-obesas. Os Fatores de Crescimento Semelhantes à Insulina I e II (Insulin-Like Growth Factors ou IGFs) são moléculas estruturalmente semelhantes à insulina, agindo na interação local e sistêmica da síntese ovariana de estrogênios. Quando não ligados aos seus receptores, são carreados pelas IGFbinding proteins $\left(\mathrm{IGFBP}_{1 \text { a } 6}\right)$. Este trabalho foi realizado no ambulatório de Endocrinologia Ginecológica do Hospital das Clinicas da Faculdade de Medicina de Ribeirão Preto - Universidade de São Paulo, de fevereiro de 1998 a março de 1999. Foram selecionadas 23 pacientes portadoras de SOP, e 21 com ciclos menstruais ovulatórios (grupos controle). Todas elas foram subdi- vididas de acordo com o seu Índice de Massa Corporal (IMC): obesas (IMC $\geq 27$ ) e não-obesas (IMC<27). Realizou-se dosagen de LH, FSH, Prolactina, hormônios andrógenos; além de testes de estímulo: Teste oral de tolerância à glicose e Teste da Levo-DOPA, obtendose as curvas glicêmica, insulínica e de $\mathrm{GH}$; e as dosagens de IGF-I e IGFBP $_{1,2} \mathrm{e}_{3}$. Todos os exames foram realizados do primeiro ao sétimo dia do ciclo menstrual nas mulheres ovulatórias, (sendo dosada Progesterona no $22^{\circ}$ dia do ciclo para se confirmar a ovulação) e em dias aleatórios nas com SOP. Realizou-se também ultra-sonografia pélvica de todas as mulheres. Os niveis de hormônios andrógenos (Testosterona, Androstenediona, Sulfato de Deidroepiandrosterona - DHEA-S e 17-OH- $\alpha-$ Progesterona) não se apresentaram fora das faixas de normalidade, porém seus valores foram mais elevados nas mulheres com SOP. Nenhuma das pacientes portadoras de SOP apresentou curva glicêmica alterada. O grupo sem obesdiade apresentou valores mais elevados em relação aos demais grupos nas curvas insulinicas. A curva de GH apresentou valores médios no grupo controle não-obeasas, e o IGF-I apresentou dosagens mais elevadas nas mulheres com SOP sem obesidade, confirmando a literatura a respeito. A associação entre RI e SOP com obesidade, e entre a melhor reserva hipofisária de $\mathrm{GH}$ e valores elevados de IGF-I e SOP sem obesidade evidencia a importância de IMC na fisiopatologia da SOP.

Palavras-chave: Síndrome dos Ovários Policísticos. Obesidade. Distúrbios menstruais. Hirsutismo. 